\title{
Las estrategias empresariales y el nuevo ambiente económico. La realidad virtual del cambio
}

\section{Paulo Peixoto de Albuquerque*}

\section{Resumen}

Este artículo analiza las estrategias de las empresas ante un entorno económico de incertidumbre que se deriva del proceso de globalización de la economía. Las innovaciones administrativas presentadas por los empresarios, expresan en realidad, un cambio "virtual" y no realmente modificaciones significativas en el proceso de organización del trabajo. En todo caso, es necesario conocer lo que ocurre de hecho en las organizaciones para no caer en la trampa de los tecnicismos administrativos.

Palabras clave: Estrategias empresariales, innovaciones tecnológicas, proceso de trabajo, asociaciones empresariales, tecnología administrativa.

\section{Management Stategies and the New Economic Environment. The Virtual Reality of Change}

\section{Abstract}

This article analyzes management strategies in an uncertain economic environment which derives from the process of economic globalization. The administrative innovations presented by private management express in reality a "virtual change" and not

\footnotetext{
Recibido: 03-04-98 . Aceptado: 05-06-98
}

- Prof. Dr. de Sociología en la Universidad do Vale do Rio dos Sinos - RS/Brasil. Dirección: Rua São José. 627 - CEP 93.040.000 - São Leopoldo. Telefax: 0515882161 . e-mail: albuq @compujob. com.br 
really significant modifications in the process of labor organization. In any case, it is necessary to understand what has really occurred organizations so as not to fall into the trap of administrative technicisms.

Key words: Management strategies, technological innovations, Labor process, business associations, administrative technology.

\section{Introducción}

Vivimos en un mundo de rápidas y profundas transformaciones que sorprenden, dejan a las personas perplejas, angustiadas, principalmente porque los cambios rompen con conceptos, usos y costumbres, no permitiendo el tiempo suficiente para la adaptación a nuevas situaciones. Es la crisis. De la misma forma, las mutaciones que están en curso en la economía, el trabajo, la vida de las personas parecen indicar que la crisis no es pasajera, por el contrario, forma parte de los nuevos tiempos.

Nuevos tiempos que insinúan una extraña mezcla de dos estados de espíritu: la sensación de partida para un mundo nuevo y una sensación de que el viejo ya no existe y que las personas están enfrentadas con una situación nueva, que exige respuestas completamente nuevas, un nuevo paradigma. El concepto de paradigma. (Thomas Kuhn, 1970) creció y ultrapasó la definición existente en los diccionarios. Actualmente el término paradigma es constantemente usado para definir un modelo amplio, un "framework", una manera de pensar, 0 un esquema de comprensión de la realidad.

Esto no es totalmente verdadero, porque existe una considerable continuidad en el modo de pensar los problemas con respecto a los avances de la tecnología que la reestructuración de las econo- mias nacionales no ha solucionado y que nosotros simplemente empujamos hacia adelante.

Los títulos de los libros nuevos de administración y negocios escritos por importantes pensadores se sitúan al nivel del relato de las condiciones de cambio en el ambiente empresarial y discuten los cambios fundamentales en la situación económica mundial y la necesidad de un nuevo paradigma de una forma simplista, que no posibilita comprender y tratar las nuevas realidades del proceso productivo.

El problema del cual estamos hablando se refiere a las mutaciones del proceso productivo y de la organización del trabajo, tema que pasó a ser tratado y focalizado a partir de un cierto particularismo normativo ligado a un proceso de globalización, que privilegia el punto de vista económico y valoriza el "consenso" como factores fundamentales de la modernización de los procesos productivos. (Barlett, 1992; Ohmae, 1990; Aburdene y Naisbit, 1990; Porter, 1990)

La preocupante realidad del nuevo ambiente productivo es el surgimiento de una nueva era en términos de competencia. Una competencia que no se realiza tan solo entre los participantes tradicionales y en mercados tradicionales, sino a partir de nuevos actores de sectores industriales o económicos, principalmente porque la desintegración de barreras de 
acceso a mercados anteriormente aislados y protegidos, pasó a ser el dato diferencial del nuevo ambiente económico. $Y$ esto exige nuevas estrategias.

En este sentido, los cambios del proceso productivo y la modernización han sido focalizados en la mayor parte de los discursos empresariales como sinónimo de prácticas consensuales que posibilitan a los actores sociales, en el proceso de trabajo, innovar y expresar sus habilidades para el alcance de objetivos organizativos.

Delante de esta nueva realidad, acuerdos y asociaciones entre empresas están siendo presentados por los medios, como la superación de formas tradicionales y antiguas de organización de la producción e indicadas como estrategias empresariales más eficaces en ambientes económicos de incertidumbre, pues presentan las virtudes del "consenso" como factor fundamental para agregar valor al trabajo.

Tal como vienen siendo presentadas, son propuestas técnico-jurídicas que sugieren la superación de formas de organización del trabajo, cuyo escenario es diseñado por el mercado y por las nuevas coyunturas del capitalismo contemporáneo.

En este sentido, "acuerdos"y "asociaciones" han venido siendo la forma por la cual la literatura técnica (Druker, 1988; Mills, 1991; Savage, 1990) torna posible una aprehensión fragmentada de lo real que agrada por su "buen sentido" y contribuye a la proliferación de modalidades de administración que confunden las tentativas de análisis más am- plias del significado de estos "acuerdos y asociaciones".

Es claro que los problemas de segmentación de lo real no invalidan estudios o "lecturas" que se hacen sobre el trabajo, sobre los procesos de organización o sobre las organizaciones. Pretender dar una validación a los cambios sin cuestionar las prácticas sociales históricamente creadas, porque los locutores convencen a sus compañeros a partir de un recetario normativo, es no considerar que las relaciones sociales que caracterizan lo cotidiano de las organizaciones son más complejas y no siempre transparentes.

Los años 90 constituyen una década importante porque señalan un momento de transición, debido a que, para hacer frente al nuevo ambiente económico, las empresas están asumiendo formas y tipos de organización de la producción que expresan otro paradigma tecnológico y de organización del trabajo.

Por otro lado, momentos de transición, como los que están siendo vivenciados, son importantes para re-ver y considerar las prácticas de organización del trabajo. Organización del trabajo que puede ser entendida como un patrón regulado y regular de interacción que es conocido y practicado (aunque no necesariamente aprobado) por los actores sociales involucrados en el proceso de trabajo, el cual tiene la expectativa de continuar interactuando sobre las normas sancionadas y garantizadas por ese patrón. (Albuquerque, 1995)

En este sentido y así definido, el sistema productivo es necesariamente una construcción social, en la medida en 
que remite las propuestas organizativas del proceso de trabajo y de sus estrategias a un conjunto de procedimientos más o menos específicos de un determinado actor social que propone y/o impone un significado a las relaciones de producción.

Es importante esclarecer este concepto, porque regularmente las nuevas estrategias empresariales son presentadas de forma que relativizan las tensiones que existen en las prácticas sociales $y$ en las acciones que intentan implementar.

En la versión técnico-administrativa de las relaciones de producción, los conceptos de "asociaciones y alianzas" coexisten sin dificultad; las diferentes implicaciones sociológicas y políticas a que uno y atro concepto remiten no emergen, porque sus pretensiones de validez no son cuestionadas por otros actores sociales.

Es interesante resaltar este aspecto, porque el capitalismo supone una organización histórica particular del monopolio de los saberes socialmente significativos; y la tecnología de organización del trabajo es uno de esos saberes que se produce y reproduce en un contexto de conflicto y antagonismo.

Se percibe en las propuestas administrativas actuales, algo de original y de idiosincrático que se expresa en la necesidad de mejorar la productividad de los trabajadores, en la capacidad de respuesta empresarial que el mercado desea, en la relativización de los antagonismos y de los conflictos dentro de las organizaciones, lo cual constituye una forma muy particular de materializar la lucha de clases.
El énfasis en procesos integradores al nivel de la tecnología de producción y consensuales en la administración de la fuerza de trabajo, recodifica la cuestión de las tensiones capital-trabajo, remitiéndolas a un doble proceso: -de globalización y fragmentación-, cuya característica principal es el no cuestionamiento de la monopolización de un tipo de conocimiento y de determinadas actitudes y la descontextualización de los hechos y de las relaciones entre diferentes socios, colaboradores y/o actores sociales.

Se percibe actualmente, en la mayor parte de las empresas, un comportamiento mas o menos uniforme, que se traduce por la adhesión al "the only game in town", o sea, la globalización de las estrategias empresariales. Se ha hablado mucho sobre la globalización, y no es nuestra intención abordar este tema, pero sí conviene resaltar algunos aspectos de dicho proceso en las empresas, en la medida en que pasan a señalar y a ser parámetros de lo que llamamos "estrategias de las empresas".

La globalización, al articular diferentes tiempos y ritmos productivos, trajo consigo una dinámica y horizontes que fragmentan la perspectiva nacional y la percepción de un futuro nacional. Esto significa la modíficación del sentido dado a la autonomía productiva, donde los tiempos y los ritmos fabriles eran determinados enteramente por las organizaciones.

Con eso no quiero decir que la globalización elimina la referencia nacional, sino que las nuevas instancias reguladoras supranacionales remiten a una redefinición del modo de organizar el proceso productivo y la actuación de las empresas (Boyer y Sailard, 1995). 
En otras palabras, no se pueden pensar los factores internos de las organizaciones o de las economías nacionales de forma aislada, porque las organizaciones y las economías nacionales se encuentran cada vez más entrelazadas con la economía global (Boyer, 1995). En este sentido, discutir las mutaciones del sistema productivo significa pensarlo:

a) en su dinámica procesal, a partir de la naturaleza específica de una nueva forma de organizar el trabajo;

b) Intentando captar su racionalidad económica, su significado productivo en cuanto pertinencia social.

Siendo así, en el ámbito de este artículo, trabajo con las siguientes hipótesis:

1) Sin una revaluación de las concepciones de acuerdo, alianzas y asociaclones sugeridas por la literatura normativa de la "administración de empresas", no se podrá afirmar que existe un nuevo modo de pensar las relaciones de producción en el interior del proceso productivo.

2) Las estrategias de acuerdos, asociaciones y alianzas que resaltan las virtudes del "consenso", son transferibles a la realidad tan solo en la forma jurídica, porque son el resultado de una negociación entre iguales sin el enfrentamiento directo de socios opuestos.

Son estrategias que no permiten una autonomía del sistema productivo porque los procedimientos adoptados en los acuerdos, asociaciones y alianzas son pensados unilateralmente por un actor social - las empresas- y se objetivan mucho más como estrategias de domina- ción y en función de una tecnología administrativa.

\section{Acuerdos, allanzas, asociaciones y fusiones: ¿relaciones de producción que se transforman?}

Las virtudes del consenso, producto de la negociación en oposición al enfrentamiento directo, están hoy colocadas como forma casi universal de relaciones en el sistema social.

La organización del trabajo tradicional, jerárquica, se encuentra con profundos problemas. La razón de eso son los presupuestos de organización del trabajo que se fundamentan en principios de tipo: "vigilar y controlar/comand and control"(que tiene sus raices en las burocracias eclesiásticas y militares de los tiempos pasados) que divide a las personas en dos grupos: los gobernados y los gobernantes y no admiten la existencia de lógicas discordantes en su interior.

Hoy, en las organizaciones, sean éstas privadas, estatales o asociativas, se percibe que el pensamiento discordante es constreñido y cede el lugar al pacto. En el interior de las empresas, esto se verifica en los discursos que acentúan el paso del valor de la jerarquia sobre el trabajo, al trabajo en equipo, a la valorización de la iniciativa de cada uno, más que la sumisión a las directivas que vienen de la dirección.

Estos hechos repercuten en las relaciones entre empresas y se objetivan en formas de colaboración y alianzas que se caracterizan por la búsqueda de socios, colaboradores entre aquellos 
que antes eran concurrentes. "Creced y multiplicaos es la estrategia de las grandes corporaciones(...); para garantizar su lugar en la globalización competitiva, las compañias están haciendo uso de fusiones $y$ adquisiciones y con eso aumentando su área de actuación" (Zero Hora, 1996)

En esta búsqueda de socios "que antes eran opuestos y estaban en competencia", se percibe que el socio no es el trabajador $y / o$ su representante-el sindicato-, sino otras empresas.

Entre 1990 y septiembre de 1996 , de 501 operaciones entre empresas en el sector de alta tecnología, el $46 \%$ fueron fusiones o absorción, y el $54 \%$ constituyeron alianzas (Ernest \& Young, 1996).

Más que una transformación de las relaciones dentro de las empresas, se asiste a la combinación de dos tipos de comportamientos:

1) Grandes operaciones de concentración empresarial que contribuyen a tornar evidente la importancia del juego de la concurrencia;

2) La ideología creciente del "consenso" que seguidamente ha conducido a la utilización casi sistemática de los términos colaboración o alianza, en la denominación de acuerdos de tipo contractual concluidos entre las empresas.

Lo que llama la atención en estas estrategias empresariales consideradas novedosas es el hecho de que los acuer- dos no substituyen la competencia por la cooperación. Por el contrario, son estrategias que tienen por objetivo permitir al conjunto de socios resistir o lanzar un ataque contra los grupos que no son miembros o iguales y, por otro lado, sirven de parámetro para el ejercicio de relaciones de influencia y de dominación entre los socios.

"la opción es que las empresas se asocien y se adapten o desaparezcan del mercado (...) la reducción del número de empresas va a crear grupos más estructurados que modificarán las relaciones comerciales (...) en ese esquema las empresas brasileñas deben asociar. se a las extrajeras para garantizar su lugar en el mercado". 1

Conviene resaltar que influencia y dominación no excluyen la cooperación, y las alianzas ${ }^{2}$. Acuerdos y asociaciones parecen marcar la transformación de la estrategia de las empresas frente a un ambiente económico de cambios e incertidumbre.

Es interesante ver que las estrategias marcadas por acuerdos y asociaciones establecen relaciones de cooperación entre determinado tipo de actor social, las empresas, pero no cualquier empresa. Estas estrategias sólo conciernen a aquellas empresas dotadas de capacidad estratégica propia, cuya especificidad está en su carácter de gran empresa

1 Material publicado en el Diario Comercio \& Industria, São Paulo, marzo de 1995. Entrevista con José Carlos Coimbra- Asesor de comercio exterior del Sindicato Nacional de Industria y Comercio de Componentes para vehículos automotores (SINDIPEÇAS).

2 El "affair" METAL LEVE empresa comprada por el consorcio COFAP/MAHLE indica que, si no hay control, el extranjero se queda con su capacidad de crecimiento limitada. 
transnacional. Los casos mas ilustres son los siguientes: Colgante/Kolynos, Philip Moris/Lacta, Grupo Abrilfolha de Manhã.

Grandes empresas cuya definición productiva y estructuración organizativa amplía el modo de pensar la producción, porque sus acciones son determinadas por la idea de mercado global. La empresa se está ampliando con base en nuevos tipos de relaciones, que las capacitan para desarrollar un abordaje más amplio del mercado.

En vista de eso, empresas que anteriormente presentaban pocos puntos en común están haciendo fusiones o formando joint-ventures para así responder rápidamente a oportunidades nuevas 0 efímeras, tener acceso a los clientes de la otra, crear nuevos mercados, compartir informaciones, actuar de forma conjunta, como grupos de intereses o lobbies, expandirse geográficamente con rapidez, etc. Son formas de organización de la producción que corresponden a otro tipo de arquitectura empresarial y que concurren para modelar un determinado tipo de funcionamiento y organización de la empresa que no se restringe a la cuestión organizativa interna de la fuerza de trabajo. ${ }^{3}$

\section{Los acuerdos y asociaciones: estrategias de adaptación al nuevo ambiente económico}

Los análisis tradicionales de las escuelas de administración reducen este complejo proceso de formación de acuerdos y asociaciones a los aspectos procesales y/o formal -jurídicos, considerándolos como formas de relaciones entre empresas que asumen lo social como equivalente de mercado; relaciones sociales entendidas apenas como instancia de intercambio y la organización como un espacio objetivo de jerarquía o de compromiso.

En vista de eso, la cuestión es saber si esta empresa mutante, ampliada o en red como resultado de acuerdos, asociaciones y fusiones, traspone horizontalmente las fronteras de la organización tradicional y puede ser considerada innovadora.

Todos los análisis de las mutaciones del sistema industrial insinúan que las transformaciones de las últimas dos décadas se deben fundamentalmente al crecimiento del papel de la tecnología y del contenido de los conocimientos, que resulta en la complejidad cada vez mayor de los productos así como de los sistemas que posibilitan su producción: elevado componente de sistemas informatizados, de comunicación y producción automatizada y un componente extremamente fuerte de programación.

las organizaciones están estableciendo nuevas normas de estructuras corporativas y, al mismo tiempo, están cambiando profundamente la manera por la cual las personas desempeñan sus funciones. La pirámide está siendo substi-

3 Son formas de organización o de arquitectura empresarial que pueden involucrar la creación de grupos de investigación y desarrollo de productos, negocios de cross-licencing y hasta asociaclones que reúnen diversas industrias que pueden ser centralizadas por un banco, como en el Japón (los keiretsu). 
tuida por una nueva forma organizacional. Tanto las áreas administrativas como las de producción están siendo organizadas en equipos de trabajo, posicionados horizontalmente con nuevas atribuciones y responsabilidades. La organización en red abierta (networking organization) está basada en equipos cooperativos y multidisciplinarios, y en unidades de negocio transversalmente interligadas en red por toda la empresa (Tapscott y Caston, 1995)

Al generalizar el papel de la informática se induce a un cierto número de consecuencias en la organización y al no funcionamiento del sistema fabril tradicional. Las empresas pasan a ser pensadas como una red de funciones en que las operaciones o negocios se interligan. En esta formación los límites entre las empresas se tornan poco nítidos y la estrategia se desarrolla en un contexto más amplio y abierto, de fuera hacia dentro, en vez de ser de dentro hacia fuera como anteriormente.

En este contexto, la fuerza de trabajo (mano de obra) se torna portátil, flexible, pudiendo ser obtenida por medio de outsourcing y por medio de la subcontratación de diversas atribuciones. La nueva tecnología capacita la integración de componentes organizacionales modulares e independientes, como en una red integrada de empresas. Pueden ser eliminadas superposiciones funcionales y definiciones no claras o conflictivas, de responsabilidades en procesos que trasponen fronteras funcionales. Los conceptos de uniformidad y transparencia son aplicables a las organizaciones, de la misma manera que lo son en la arquitectura de las computadoras.

En función de esta estructura, las empresas pasan a trabajar en tiempo real, esto es, están continuamente ajustándose a los cambios ocurridos en las condiciones del mercado. Los sistemas de información y de automatización de la producción, en este sentido, son fundamentales para la captación en línea de informaciones y la actualización de los bancos de datos en tiempo real, ofreciendo una visión bastante precisa o tornando posible la gerencia segundo a segundo de algún proceso productivo.

El proceso productivo no estando condicionado a una estructura rígida, puede ser rápidamente adaptado a la fabricación de series limitadas para mercados emergentes o para segmentos importantes, considerados nichos significativos de mercado. Así, competir en el nuevo ambiente económico implica una redefinición de tiempo y espacio para funcionarios y partes interesadas; significa substituir las estructuras rigidas por una arquitectura organizacional modular en la cual los tiempos de negocio operan a partir de funciones marcadas por el concepto: cliente-abastecedor.

En este tipo de empresa en red, cualquier persona o equipo puede comunicarse y, conforme sea apropiado, compartir informaciones con cualquier otra empresa o equipo. El trabajo puede ser ejecutado desde diversos puntos. La red se torna en la depositaria de comunicaciones independientemente del tiempo, para personas que accesan a comunicaciones recibidas de otros.

La empresa se transforma en una infra-estructura o base de cooperación. 
Si personas y grupos pueden actuar como clientes y abastecedores, cooperando unos con los otros, no en función de principios morales, sino en función de sus propios y múltiples intereses, entonces la noción de cooperación puede ser ampliada, más allá de las relaciones existentes entre la mano de obra y la administración, tornándose el modus operandi de la empresa.

Modus operandi que legitima un tipo de estrategias (acuerdos y asociaciones) los cuales a su vez establecen dos niveles de interdependencias: a) entre diversas partes de la empresa y b) entre empresas que son redefinidas e incorporadas a la nueva infra-estructura productiva por la noción de producto dirigido hacia el mercado. (Aburdene y Naisbit, 1990). Esta mutación de la arquitectura organizacional va de la mano con otra concepción de lo que es producto. La noción substantiva de producto se transforma y es substituida por su adjetivo performance, en la medida en que el foco central del trabajo es adaptar cada vez más la producción a las necesidades de la clientela.

Hoy, una oferta de "performance"y no de productos, expresa en el lenguaje común, algunos significados que conviene resaltar porque indican mutaciones del sistema industrial caracterizado por el crecimiento del papel del conocimiento, resultando en 10 que es comúnmente considerado como un cambio del paradigma tecnológico (Dosi, 1984; Camagni, 1988; Coriat, 1990).

La nueva empresa da énfasis al trabajador del conocimiento (knowledge worker). Por definición, el conocimiento requiere especialización. Como ocurre con la tecnología que es especializada para poder atender a requisitos específi$\cos$, las competencias-asignaciones especializadas son estimuladas y desarrolladas en personas y en equipos.

La nueva tecnología redefine tiempo y espacio para sus funcionarios y partes interesadas, lo que resulta en la substitución de las relaciones sociales de producción tensionantes por la idea de proveedores, productores y clientes.

En este sentido, la empresa en forma de red intenta ser una organización abierta que une sus miembros en torno de una visión con la cual las personas están verdaderamente comprometidas. Las actividades de trabajo individual son reestructuradas de tal forma que cada individuo pasa la mayor parte de su tiempo desempeñando la actividad que se constituye en su responsabilidad primaria.

El nuevo paradigma es lógicamente fundamental en la generalización y difusión de la informatización en el conjunto del sistema de producción. Este se apoya en las tecnologías susceptibles de servir de base para amplios espacios de aplicación.

El nuevo paradigma permite las condiciones para la flexibilidad máxima de tiempo y espacio en los sistemas industriales, marcado por la innovación continua en la reducción consecutiva del ciclo de vida de los productos y en una intensa circulación de informaciones. En este sentido, el intercambio de bienes se transforma en un caso de cooperación donde la competencia del vendedor y del comprador se interpenetran para la elaboración de un producto que todos esperan sea producido. 


\section{Acuerdos y asociaciones en la industria, un tipo de relación que necesita ser explicitado}

Los acuerdos y asociaciones, al aplicar una coordinación entre operaciones de empresas socias, ponen en cuestión la transformación de los modos de funcionamiento de la organización industrial. La generalización de las operaciones asociadas, influencia y altera las formas de concurrencia, al mismo tiempo que provoca ciertas mutaciones en las estructuras industriales. Se asiste a una homogenización creciente de productos y de procesos de producción. Las empresas aceleran sus movimientos de multinacionalización y aplican en cada lugar sistemas mundiales de concepción, de fabricación y de comercialización.

Así, la empresa globalizada y colocada en contacto con empresas que poseen sistemas productivos distintos, para viabilizar la asociación, necesitan de la multiplicación de las formas de concurrencia y de una exacerbación de la competencia mundial. La globalización facilita la conclusión de acuerdos de cooperación en la medida en que la extensión del paradigma tecnológico de redes (networking company) en escala planetaria aproxima los modos de funcionamiento de las empresas, permitiendo la organización de operaciones llevadas conjuntamente de modo más eficaz. Pero todavía más, ésta incita a la conclusión de acuerdos porque coloca en relación empresas de potencias comparables, que ocupan posiciones sólidas en su ambiente doméstico.

La paradoja es que la homogenización es al mismo tiempo una multiplicidad que torna posible y justifica el recurso de las estrategias de cooperación, porque se transformó la noción de producto y el modo de relacionarse con la clientela.

Insistimos anteriormente en la transformación de las relaciones de oferta en que la empresa pasa de abastecedor de productos a una oferta de performance. La generalización de esa medida implica una interpenetración de competencias entre productores y utilizadores por la concepción de bienes adaptados a caracteristicas de utilización específicas. La cooperación es seguidamente un modo de comercialización necesario.

En términos de concurrencia, los acuerdos y asociaciones concluidos tienen por objetivo conquistar a la clientela en un periodo de innovación rápida y de aparición continua de nuevos productos, al mismo tiempo que insinúan que las actuales formas de organización del mercado son limitadas.

La internacionalización, abriendo los espacios del mercado-nación, refuerza el impacto de la concurrencia e incita al cierre de acuerdos. Seguidamente las empresas nacionales forman alianzas con sus homólogas para mantener una cierta capacidad nacional de producción y así colocar más fácilmente los productos en el mercado, sean estos productos venidos del exterior a través de la importación o por la implantación de filiales.

Lo interesante a resaltar en estas estrategias empresariales es que, paralelamente al establecimiento de acuerdos y asociaciones, se nota la aceleración de alianzas interindustriales de empresas no directamente concurrentes. Este hecho resalta el aspecto mutante de la nueva arquitectura organizacional- la estructura- 
ción de empresas en redes- donde la naturaleza combinatoria de las actividades industriales se va a manifestar en la modularidad del equipo, de los productos y en la multiplicidad de las soluciones de oferta, no solamente de productos, sino de producción de performance.

Así, los productores más especializados se posicionan en nichos de mercado $y$ ofrecen productos que suponen la integración de producto-sistema, o sea, una tecnología productiva de punta. Pero, como el dominio de la tecnología en Brasil, igual que en la mayor parte del sector industrial de América Latina, se caracteriza por una fuerte dispersión de los mercados y los productores, las opciones estratégicas de las empresas brasileñas se reducen a intentar:

1. La fusión con la industria mundial estandarizada que corresponde a la estrategia dominante de los principales grupos brasileños. Esta se apoya sobre una idea de globalización de la industria, la transnacionalización de los mercados y en términos económicos a partir de la banalización de los productos.

2. La participación y estructuración de un espacio latinoamericano abierto al comercio que se concretiza por la formación de bloques económicos que acompañan la lógica mundial; el Mercosur es un ejemplo de esta estrategia.

La barrera territorial, que podría ser un factor de bloqueo, es bordeada por los grupos suficientemente fuertes implantados a nivel mundial, que relativizan estas barreras territoriales. Así, esta lógica consiste en observar los mercados más importantes e implantarse lo más rápidamente posible a través de estrategias que no provoquen conflicto o resistencia.
La multiplicación de los acuerdos de cooperación entre firmas europeas o americanas y firmas brasileñas resultó en la repartición del mercado brasileño y una transformación del sistema industrial, principalmente por la noción de que el proceso de organización del trabajo debe ser pensado como profundización de las relaciones entre abastecedores-productores y clientes a partir de la idea de "producción-performance", que pone en cuestión el estatus actual de la concepción de producto y por consecuencia, aquello que se entiende por mercado, sin provocar conflictos o antagonismos.

En este sentido, la transversalidad de la tecnología en el centro del nuevo paradigma tecnológico, participa de la revisión de los sistemas verticalizados de organización de la producción y de la fuerza de trabajo e implica la necesidad de trabajar dentro de una perspectiva que valoriza el consenso, asegurando una interpenetración de las actividades industriales.

\section{Conclusiones}

\section{Repensando la estrategia empresarial de los acuerdos y la valorización del consenso}

De manera general, el interés analítico de los acuerdos no reside en el estudio de los acuerdos por sí mismos, sino en lo que ellos pueden aportar para la comprensión de los modos de relación entre empresas.

El marco de referencia de análisis de los acuerdos debe ser aquel de la estrategia de los actores sociales y no únicamente la estrategia empresarial de ciertas empresas. 
Siendo así, el cambio del proceso productivo pasa a ser no sólo de los factores de producción por la utilización más intensa de nuevas tecnologías, también hay que repensar como los actores sociales, socios del proceso de trabajo, piensan organizarse con el fin de alcanzar resultados y engendrar acciones y resultados aceptables para todos los individuos y grupos.

Más que adaptarse a los cambios de un ambiente económico inestable e incierto, se percibe que las estrategias empresariales, principalmente de los grandes grupos que se insertan en una cooperación tienen por objetivo:

- primero, resaltar un tipo de conocimiento (técnico) que se valoriza por su transversalidad y aplicación en diferentes espacios productivos;

- segundo, establecer un determinado tipo de evolución, protegiendo sus actividades en el futuro así como protegen sus inversiones contra el brutal aparecimiento de nuevas opciones técnicas.

En este sentido, no se puede decir que las alteraciones de la estructura organizacional modifican significativamente las relaciones sociales dentro de las empresas. En la literatura de administración de empresas, preocupada por la gerencia, queda bien explicitado que los acuerdos de cooperación aparecen más como un intento de control de un actor social- las empresas- particularmente apropiada para ambientes de incertidumbre económica.

La actualidad de estas estrategias deriva directamente de prácticas que van más adelante del simple juego de complementariedad donde cada uno de los socios aporta un elemento particular, una pieza al rompecabezas como en las operaciones tradicionales de tercerización.

Ciertamente las exigencias de rapidez e igualmente de sofisticación creciente de los componentes elementales de los productos conducen cada vez más a la transformación de los contratos de tercerización en verdaderas relaciones de asociación donde ejecutante y demandante participan de procedimientos y de afinación de productos, instaurando una verdadera concertación técnica de coproductores (Coriat, 1990).

Entre tanto, conviene destacar que en Brasil la generalización de nuevas formas de acuerdo del tipo asociación, alianzas, no substituye las formas tradicionales de control de la fuerza de trabajo. Los cambios de tecnología contra algún sector del mercado y los acuerdos de complementariedad, son estrategias tradicionales que están y continúan presentes en la actuación empresarial y como tal son utilizadas; no teniendo nada que ver con la forma como se objetivan las relaciones sociales en el interior del proceso productivo.

El aspecto tradicional se explicita en la referencia a los costos de producción que domina una buena parte de la literatura sobre los acuerdos entre empresas (Albrecht, 1992). Este descansa en la idea de que las instituciones del sistema capitalista tienen por principal fin y resultado la economía en los costos de producción (Williamson, 1995).

Las empresas confrontadas con la inestabilidad del mercado actual, sea interno o externo, tienen costos de transacción cada vez más altos, dado que la flexibilidad del mercado no se corresponde con la rigidez interna de las relaciones de 
producción en serie, basada en un modo de funcionamiento de tipo jerárquico.

En este sentido, los acuerdos contractuales se constituyen entonces en la estrategia que busca evidenciar el consenso como valor capaz de mediar jurídicamente la discordancia existente entre un mercado flexible e inestable y la organización que aún conserva características de rigidez.

Este abordaje jurídico que sirve para atenuar el costo vinculado a las transacciones comerciales, en primer lugar, está ligado a un carácter más descriptivo que explicativo, según permite justificar la eficacia de una forma de organización, porque atenuar el costo ligado a transacciones comerciales es diferente a controlar el costo de transacciones sociales inherentes a un modo de organizar el proceso productivo y pensar la organización de la fuerza de trabajo.

Una investigación previa sobre reconversión industrial y nuevas formas de gestión de la fuerza de trabajo (Albuquerque, 1995) indicó que los hechos que constituyen las "estrategias de acuerdo no tienen la reducción de los costos como un elemento motor. El estudio más sistemático sobre la racionalidad de las formas de cooperación, indica un aspecto fuera del mercado, señalando que los cambios en la organización y control del trabajo provocados por la reconversión del proceso productivo y por las estrategias empresariales expresan:

1. Una concepción de desarrollo determinada desde fuera, en la cual el espacio de autonomía de las empresas variará según la forma de inserción (o no) en el sistema económico internacional; parafraseando a Leiptz, se po- dría clasificar de "neofordismo periférico"esa concepción de desarrollo determinado por el exterior.

2. La necesidad de que se integren en el sistema de poder (Estado) las demandas especificas de un actor social en términos económicos o sociales capaces de influenciar directa o indirectamente la política industrial (Petrella, 1989);

3. La preocupación de las empresas brasileñas por integrarse al sistema económico mundial;

4. La composición de competencias (en cuanto calificación) a través de acuerdos donde la incapacidad tecnológica de uno es compensada por la tecnologia del otro, es considerada the only best way" para el desarrollo, presentándolo como un hecho derivado de la concertación social;

5. Las innovaciones sociales del proceso de trabajo y de la arquitectura organizacional son presentadas como prueba visible de las transformaciones económicas y sociales.

Se percibe que los elementos centrales de las estrategias empresariales pueden ser externalizados en los acuerdos y asociaciones entre firmas, pero lo que realmente se verifica es que este recentrar puede ir hasta la constitución de firmas virtuales (the hollow corporations) donde solamente la competencia logistica y el saber comercial son consenados por la empresa que terceriza todas las otras actividades.

El término "extended company" es apropiado en la medida en que este hace referencia a conjuntos de firmas que se reagrupan con socios exteriores diferen- 
tes, ligados a objetivos contractuales comunes en torno de una sociedad central.

La reducción de costos burocráticos o costos de gestión pueden ser incluidos como motivo de tercerización, pero es difícil sustentar que estos puedan ser superiores a los costos de coordinación de un conjunto de firmas independientes.

La justificación de la descentralización reside más en la necesidad de reducir el tiempo de acceso al mercado y mejorar la adecuación de la oferta a determinados segmentos estrechos de la demanda.

La "empresa en red" funciona en paralelo y no en modo secuencial; este tipo de organización refuerza el agrupamiento de empresas especializadas complementarias, $y$, al hacerlo incremen ta la especialización de cada uno de los participantes. En este caso, la firma central continúa siendo la gestora principal del conjunto y decide quién y qué se terceriza y cuáles los resultados esperados.

En este sentido, el clivaje de la descentralización no conduce a una alteración substancial de la lógica de recorte tayloriano del proceso de producción, dado que el fraccionamiento de la empresa en un conjunto de entidades autónomas involucradas en una red de cooperación no niega el eje de la concepción taylorista.

Es preciso tener presente que los acuerdos y asociaciones desde el punto de vista de la estrategia de las empresas no se limitan a la consideración del acuerdo como un objeto único, materializando su ligazón entre socios, si no como parte de sus actividades y como elemento sometido a su lógica propia. Es importante tener presente que las alianzas entre gru- pos constituyen un marco estratégico orientado no a los resultados sino a acciones.

El análisis de las cooperaciones pone en evidencia que la complementariedad de los socios se da sobre una base de fragilidad, porque está fundada en una asociación vertical equivalente a un intercambio de capacidades que se adicionan y no de integración. Cada una de las em. presas posee uno o más activos específicos y buscará asociarse mientras continúe controlando el mercado donde los productos puedan ser presentados. Son estrategias que no permiten una autonomia del sistema productivo, porque las limitaciones del nuevo paradigma tecnológico condicionan claramente la orientación de las estrategias del acuerdo.

Se constata que una buena parte de la aceleración del fenómeno se inscribe en una lógica general de relaciones sociales unilaterales entre agentes económicos iguales que subestiman los demás actores económicos y sociales: el Estado y los sindicatos.

En este sentido, reiteramos, existe algo de verdadero y algo de falacioso en estas estrategias empresariales; verdadero, porque la globalización trajo consigo una revalorización del consenso, y falacioso, porque las innovaciones propuestas por estas estrategias son hechas entre aquellos que siempre fueron socios.

El hecho nuevo deriva de la retirada de dos actores sociales del proceso productivo, el sindicato afectado por una "anomia sindical" que imposibilita acciones de contrapunto y la retirada del Estado, legitimada política e ideológicamente por el neoliberalismo. Evidentemente, 
"usted no necesita ser un meteorologista para saber la dirección del viento" (Bob Dylan).

En verdad, estas estrategias empresariales señalizan la pérdida de capacidades y la relativización del saber operario substituido por la noción de especialización de conocimientos o construcción de capacidades, evidenciada en los numerosos acuerdos y asociaciones que indican que estas se constituyen en mucho más que simples combinaciones de adaptación del espacio industrial entre los productores para levantar barreras a la entrada de nuevas corrientes; sino es una modalidad de concurrencia que favorece a los grandes grupos que se esfuerzan por controlar y orientar el proceso económico y neutralizar la posibilidad de acción de otros actores sociales.

En este sentido, pensar en acuerdos y asociaciones nos remite al imaginario social que, dependiendo de "donde se habla", puede tener otros significados, como muy bien traduce el diálogo de Alice y Humpty Dumpty.

". Cuando uso una palabra -dice Humpty Dumpty en tono irónico- esta significa exactamente aquello que yo quiero que signifique...ni más ni menos.

- La cuestión, ponderó Alice -es saber si el señor puede hacer que las palabras digan cosas diferentes-

- La cuestión -replicó Humpty Dumpty- es saber quién es quién manda sólo eso."

\section{Referencias bibliográficas}

Aburdene, Patricia \& Naisbit, John (1985). Reinventing the corporation: Transforming your job and your compa- ny for the new informations society. New York, Warner Books.

Aburdene, Patricia \& Naisbit, John (1990). Megatrends 2000, Tem new directions for the 1990, New York, Willina Morrow and Company.

Albrech, Karl (1993). A única coisa que ìmporta: trazendo o cliente para dentro de sua empresa. Săo Paulo, Pioneía.

Albuquerque, Paulo P. (1995). Reconversion industrielle et les nouvelles formes de gestion de la force de travail, tese de doutorado, Universite Catholique de Louvain-la-Neuve, Belgica.

Barlett, Donald L. \& Steele, James B. (1992). America: what went wrong?, Kansas City: Andrews \& McMeel.

Boyer, R. \& Sailardy (1995). Théorie de la régulation L' Etat des davoirs. Paris, Edition La Découverte.

Boyer, ค. (1995). States against markets, the limits of globalization. Edition Routledge.

Camagni, R. (1988). Cooperative agreements and new forms of external development of companies, MODULI: Centro Studi IBM, Italia.

Coriat, B. (1990). L'atelier et les robot. Paris, C. Bourgois.

Diário Comércio \& Indústria, São Paulo, março. 1995. Entrevista con José Carlos Coimbra.

Dosi, G. (1985). Technical change and industrial transformation, London, MacMilan.

Drucker, Peter F. (1988). The New Organization. Harvard Business Review, janeiro-fevereiro.

Emest \& Young (1996). Relatório de pesquisa. Operaçōes entre Empresas, São Paulo. 
Kuhn, Thomas (1970). The structure of Scientific Revolutions. 2 " ed.. Chicago: University of Chicago Press.

Mills, D. Quinn (1991). Rebirth of the Corporation. New York: John Wiley \& Sons.

Onmae, Kenichl (1990). The Borderless World, Power and Strategy in the Interlinked Economy". New York: Harper Collins Publisher.

Petrella, R. (1989) La mondialisation de la technologie et de l'économie. Une hypo thése prospective. FUTURIBLES, \# 135 , septembre.
Porter, Michael E. (1990). The competitive Advantage of Nation. New York: The Free Press.

Savage, Charles M. (1990). $5^{\text {th }}$ Generation Management: Integration Enterprises throug Human Networking New York: Digital Press.

Tapscatt, Don \& Caston, Art. (1995). Mudança de Paradigma - A Nova Promessa da Tecnologia de Informą̧ão. São Paulo: McGrawHIII/Makron.

Williamson, E. (1985). The economic institutions of capitalism. New York: The Free Press.

Zero Hora, Seção Economia, Porto Alegre, outubro 96. 